\title{
What Do We Mean When We Talk about the Linac Isocenter?
}

\author{
Mutian Zhang1, Su-Min Zhou1, Tanxia $\mathbf{Q u}{ }^{2}$ \\ ${ }^{1}$ Department of Radiation Oncology, University of Nebraska Medical Center, Omaha, USA \\ ${ }^{2}$ Isocenter Lab, New City, USA \\ Email: mutianzhang@unmc.edu
}

Received 30 June 2015; accepted 1 August 2015; published 4 August 2015

Copyright (C) 2015 by authors and Scientific Research Publishing Inc.

This work is licensed under the Creative Commons Attribution International License (CC BY). http://creativecommons.org/licenses/by/4.0/

(c) (i) Open Access

\begin{abstract}
Purpose: The isocenter of a medical linac system is a frequently used concept in clinical practice. However, so far not all the isocenters are rigorously defined. This work is intended as an attempt of deriving consistent and operable isocenter definitions. Methods: The isocenter definition is based on a fundamental concept, the axis of rotation of a rigid body. The axis of rotation is determined using the trajectory of any point on a plane that intersects the rigid body. A point on the axis of rotation is found through the minimal bounding sphere of the trajectory when the rigid body makes a full rotation. The essential mathematical tool of the isocenter definition system is three-dimensional coordinate transformation. Results: The axes of rotation of the linac collimator, gantry, and couch are established first. The linac mechanical isocenter (linac isocenter) is defined as the center of a circle that best fits the trajectory of a select linac X-ray source position. The axis of rotation and the minimal bounding sphere are cornerstones for the rotation isocenters of the collimator, gantry and couch. The definition of radiation isocenter incorporates a surrogate of the useful beam axis. Conclusions: A framework of isocenter definitions for medical linacs is presented in this manuscript. Consistent meanings of the mechanical and radiation isocenters can be achieved using this approach.
\end{abstract}

\section{Keywords}

Medical Linear Accelerator, Isocenter, Linac Isocenter, Rotation Isocenter, Radiation Isocenter, Axis of Rotation, Definition

\section{Introduction}

This work is motivated by our experience as clinical medical physicists in that we constantly encounter a partic-

How to cite this paper: Zhang, M.T., Zhou, S.-M. and Qu, T.X. (2015) What Do We Mean When We Talk about the Linac Isocenter? International Journal of Medical Physics, Clinical Engineering and Radiation Oncology, 4, 233-242.

http://dx.doi.org/10.4236/ijmpcero.2015.43028 
ular term known as the "isocenter". Being a unique concept attributed to a medical linear accelerator (linac), isocenter is used on countless occasions, and in combinations such as mechanical isocenter, radiation isocenter, rotation isocenter, imaging isocenter, treatment isocenter, and so on. These expressions seem to be self-evident - medical physicists intuitively understand their meaning and use them with few obstacles. The aforementioned isocenters are expected to coincide [1], though in reality this can hardly be true. In the past, isocenter discrepancy was not concerning for conventional radiotherapy treatments because the discrepancy is usually much smaller than the setup errors. In the recent years, stereotactic radiosurgery (SRS) with image guidance is increasingly performed on linac platforms and requires more stringent mechanical tolerances [2]. It is especially worrisome when the reported isocenter "walk-out" is often comparable to or exceeds the discrepancies between the imaging isocenter and the radiation isocenter [3].

Medical physicists are familiar with the following scenario. Prior to frame-based stereotactic radiosurgery, a ball bearing phantom representing the treatment target is mounted on the couch and aligned to the room lasers, which have been brought to a mechanical isocenter determined by means such as a frontpointer. The target alignment as a function of couch and gantry angles is verified with multiple collimated beams [4]. If misalignment is identified, the target is moved closer to the so-called radiation isocenter and subsequently, room lasers are brought to the adjusted target position for patient localization. In this scenario, the lasers are surrogates of the treatment target and radiation isocenter. In general, the target is supposed to align with the radiation isocenter. This observation leads us to wonder: in this procedure, which is the primary reference and which is to be consequently determined? We realize that such kinds of confusion result from the fact that the concept of isocenter has not been clearly defined. In the literature, an isocenter has multifold meaning. It can be a virtual point as in "linac isocenter," or a sphere when physicists check the "rotation isocenter" during annual quality assurance (QA) tests [5]. One isocenter may belong to the entire medical linac system, while another belongs to a subsystem (collimator, gantry, or couch) of the linac. Very often, determination of an isocenter is dependent on the measurement techniques [6]. Consequently, the discrepancies in isocenter positions measured by different investigators might be comparable to the magnitudes of setup errors in SRS procedures. With more and more patients undergoing linac-based SRS, the necessity of consistent isocenter definition and accurate isocenter measurement has become more prominent than ever.

In a popular radiotherapy physics textbook, the definition of linac mechanical isocenter initially was, "Mechanical isocenter is the intersection point of the axis of rotation of the collimator and the axis of rotation of the gantry [7]." This definition assumes that the two axes of rotation intersect regardless of the location of the intersection point. In reality, however, the collimator rotation axis may not intersect with the gantry rotation axis (Figure 1(a)); or the axes intersect but are not perpendicular to each other (Figure 1(b)). In a later edition of the textbook, the definition was mentioned in the statement, “... (the source-to-axis distance) is the distance from the source to axis of gantry rotation known as isocenter [8].” This definition is not a purely mechanical concept, because the radiation source is now integrated. The State of Nebraska regulations move even further by the following definition, "Isocenter means the center of the sphere through which the useful beam axis passes while the gantry moves through its full range of motions"”.

The American Association of Physicists in Medicine (AAPM) has published Reports of Task Group No. 40 (TG-40) [5] and No. 142 (TG-142) [2], to provide guidelines for the QA of medical accelerators. Surprisingly, in these documents the AAPM does not explicitly define the linac isocenters, although the rotation isocenters and coincidence of axes and isocenters are among the annual QA tests. When preparing this manuscript, we surveyed several qualified medical physicists, asking them to share their insights of the linac isocenter. Our question was, "TG-40 recommends the annual QA of coincidence of collimetry, gantry and couch axes with isocenter. How do you implement a test that is able to verify your linac is within the tolerance?” Our colleagues kindly shared their experience; from the survey we learned that the linac isocenter is intuitively understood, and usually physicists observe the coincidence of the collimator, gantry, and couch axes rather than the coincidence of the axes with the linac isocenter. Let us outline the approach one author, M.Z., takes to perform the coincidence test on a Siemens Oncor linac (Siemens Healthcare, Germany): 1) Mount a frontpointer, rotate the collimator to locate and mark a point on its tip that has minimum walk-out; 2) Rotate the gantry and use lasers as reference to adjust the frontpointer length so that the marked point can represent the “isocenter”; 3) Tape a sharp object on the couch so that its tip is as close to the marked point as possible without touching the frontpointer when the gantry rotates; 4) Rotate the gantry, collimator, and couch to observe if the coincidence of their axes is within

\footnotetext{
${ }^{1}$ http://www.sos.ne.gov/rules-and-regs/regsearch/Rules/Health_and_Human_Services_System/Title-180/Chapter-20.pdf.
} 


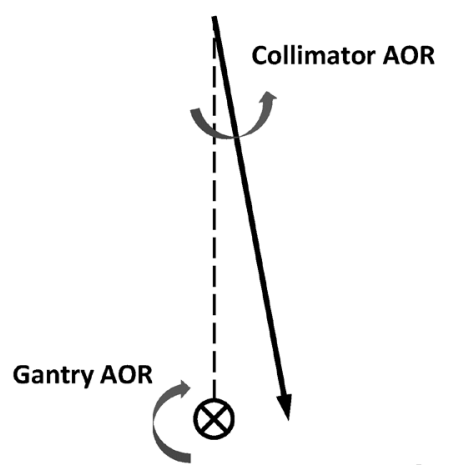

(a)

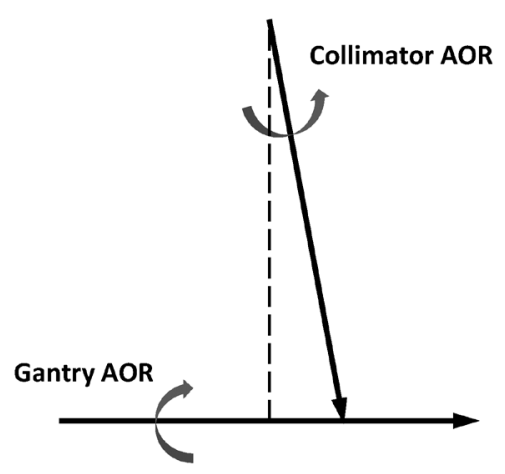

(b)

Figure 1. Two scenarios that the axis of rotation (AOR) of the collimator does not perfectly align with the AOR of the gantry. (a) The collimator AOR does not intersect with the gantry AOR. (b) The collimator AOR intersects with the gantry AOR but not perpendicularly. The dashed lines show the situation that the two axes of rotation intersect at right angle, which is the desired configuration in a medical linac.

tolerance. We must admit that in this approach, a few assumptions are implicitly made and quantification is rather difficult. The validity of the isocenter determined in step 2) is an open question. The QA results depend in large on visual observations and subjective judgment. The above QA approach is an extremely simplified version of the acceptance testing of medical linacs described in the literature. Although the linac installers have increasingly precise equipment, they still follow trial-and-error approaches. In general, they initially install and adjust mechanical components, and secondly turn on the radiation to calibrate beam collimation. The linac installation and acceptance test help us establish the grounds and logic of the isocenter definitions.

Numerous methods have been developed for the verification of linac isocenters [6]. These methods were intended mainly for detecting mechanical isocenters [9]-[11], or for verification of the radiation isocenter [12][14]. Without a consistent terminology system, however, it is difficult to interpret and compare individual studies and implement those results in clinical applications. In the next sections, we will follow the footprints of previous authors and propose a hierarchical structure of isocenter definitions. The purpose of our work is to provide the medical physics community with an example of framework in which all the isocenter measurements can be expressed using consistent terminologies. The subjects of the present study are restricted to ordinary medical linacs consisting of collimator, gantry, and couch subsystems. It is our intention to eliminate the uncertainties associated with subjective judgment and trial-and-error approaches.

The proposed isocenter definitions are based on the concept of axis of rotation. We shall make the isocenter definitions self-consistent, operable, and in addition, independent of measurement techniques. Our definition system observes three criteria: 1) It avoids circular definitions that have occurred in the literature but not clearly identified; 2) the definitions are compatible with the terminologies in the AAPM TG-40 and TG-142 reports; and 3) the definitions are mathematically operable and their physical measurements are feasible. Though our proposal does not include a specific approach of isocenter determination, we believe that once rigorous isocenter definitions are agreed upon, equivalent methods can be developed for isocenter measurement and verification. Accompanying the isocenter definitions, a mathematical model will be presented in the Methods section. This mathematical model can be used to deduce or calculate isocenter locations from the fundamental definition, or to transform the coordinates of a given point among different systems. The isocenter definitions will be presented in the Results, and the clinical applications of the isocenter concepts are addressed in the Discussion.

\section{Methods}

In this section, we present the fundamental concepts and mathematical methods that are essential to the deduction of isocenters. These are considered the minimally sufficient tools for the proposed definition system.

\subsection{Axis of Rotation}

The linac subsystems—collimator, gantry, and couch—are considered rigid bodies. When a rigid body rotates, 
every point in the rigid body rotates relative to other points. To determine the axis of rotation (AOR) of a subsystem, its rotation should be observed in a coordinate system that is stationary relative to the linac vault. Separate coordinate systems can be established on the collimator, gantry, and couch that rotate with these subsystems. The AOR definition is modified from the concept proposed by previous authors [11] [15]: The AOR is a union of points on arbitrary planes that intercept a rigid body; when the rigid body rotates, the trajectory of one such point as measured in the stationary coordinate system has the minimal bounding sphere among all the points on the same plane. Several algorithms of finding the minimal bounding sphere have been developed [16] [17]. Later in this manuscript, the concept of minimal bounding sphere will be repeatedly used in the isocenter definitions. Figure 2 illustrates the AOR definition and one approach to find a point on the AOR. Note that the trajectories might be highly irregular as opposed to the ovals in the figure.

As illustrated by Figure 2, in the reference coordinate system point $O$ is stationary, and the trajectory of an arbitrarily selected point $P$ can be accurately measured. We assume that the observer can only measure the trajectories of a limited number of points. Therefore, before the AOR is determined, point $C$ is unknown. In order to find point $C$ that is located on the same arbitrary plane addressed in the AOR definition (note that this plane is attached to the subsystem), the observer would firstly plot the trajectory of point $P$ when the subsystem finishes a full rotation. Then another point $C^{\prime}$ (not shown) is chosen on the plane. At a nominal rotation angle, vector $\boldsymbol{C}^{\prime} \boldsymbol{P}$ is calculated using $\boldsymbol{C}^{\prime} \boldsymbol{P}=\boldsymbol{O P}-\boldsymbol{O} \boldsymbol{C}^{\prime}$. The trajectory of $C^{\prime}$ is calculated based on the fact that in the subsystem, the trajectory of $P$ is a circle relative to point $C^{\prime}$. By definition, the trajectory of point $C$ on the AOR has the minimal bounding sphere. Thus the position of point $C$ can be found by global search.

\subsection{Coordinate Transformation}

This subsection presents a mathematical tool, the transformation between two coordinate systems. The coordinate systems of the collimator, gantry, and couch rotate with these subsystems. Because of the imperfection of the mechanical systems, the rotation of these coordinate systems may not follow the nominal rotation angle and can only be measured as a function of the nominal rotation angle. For simplicity, the $x, y$, and $z$ axes of these coordinate systems at the $0^{\circ}$ nominal rotation angle are set to be parallel to that of the reference system. The coordinates of a point in the collimator, gantry, and couch systems are denoted by subscripts $C, G$ and $T$, respectively. For instance, assuming that at a nominal gantry rotation angle $\theta_{G}$, the origin of the gantry system has coordinate offsets $\left(G_{x}, G_{y}, G_{z}\right)$ in the reference system, and the gantry system has rotation angles $\left(\alpha_{G}, \beta_{G}\right.$, $\gamma_{G}$ ) with respect to the $x, y$, and $z$ axes of the reference system. The direction of a rotation angle is defined in such a way that the rotation of a positive angle obeys the right-hand rule [18]. Note that these coordinates and angles are functions of $\theta_{G}$, and they are assumed to be measureable. Using the methods that were previously reported [18] [19], the following formulas are derived.

For a point with coordinates $\left(x_{G}, y_{G}, z_{G}\right)$ in the gantry system, the transformation to the reference system is

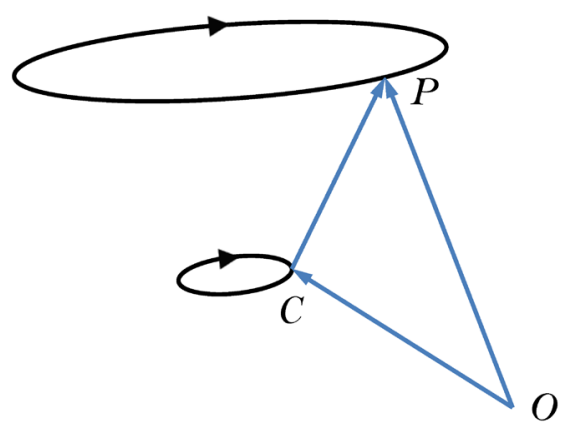

Figure 2. The trajectories of a point $C$ on the AOR and an arbitrary point $P$ on the rigid body. The two points are located on fixed positions relative to a subsystem. Observation is made within the stationary coordinate system, which has a reference point $O$. Thus the trajectory of $P$ is a roulette with regard to the trajectory of point $C$. 


$$
\left[\begin{array}{c}
x \\
y \\
z
\end{array}\right]=\left[\begin{array}{lll}
m_{G 11} & m_{G 12} & m_{G 13} \\
m_{G 21} & m_{G 22} & m_{G 23} \\
m_{G 31} & m_{G 32} & m_{G 33}
\end{array}\right]\left[\begin{array}{c}
x_{G} \\
y_{G} \\
z_{G}
\end{array}\right]+\left[\begin{array}{c}
G_{x} \\
G_{y} \\
G_{z}
\end{array}\right] .
$$

The elements in the transformation matrix $M_{G}$ are

$$
\left\{\begin{array}{l}
m_{G 11}=\cos \beta_{G} \cos \gamma_{G} \\
m_{G 12}=-\cos \beta_{G} \sin \gamma_{G} \\
m_{G 13}=\sin \beta_{G} \\
m_{G 21}=\cos \alpha_{G} \sin \gamma_{G}+\sin \alpha_{G} \sin \beta_{G} \cos \gamma_{G} \\
m_{G 22}=\cos \alpha_{G} \cos \gamma_{G}-\sin \alpha_{G} \sin \beta_{G} \sin \gamma_{G} . \\
m_{G 23}=-\sin \alpha_{G} \cos \beta_{G} \\
m_{G 31}=\sin \alpha_{G} \sin \gamma_{G}-\cos \alpha_{G} \sin \beta_{G} \cos \gamma_{G} \\
m_{G 32}=\sin \alpha_{G} \cos \gamma_{G}+\cos \alpha_{G} \sin \beta_{G} \sin \gamma_{G} \\
m_{G 33}=\cos \alpha_{G} \cos \beta_{G}
\end{array}\right.
$$

Equations (1) and (2) differ from the regular coordinate transformations in that the subsystems do not necessarily rotate about fixed origins relative to the stationary reference system. The linac gantry and couch have independent AOR; however, the collimator AOR is a function of the gantry angle. To simplify the mathematical treatment, we use the first-order approximation by assuming that the collimator AOR trajectory is not a function of gantry angle. The transformation matrix $M_{C}$ and coordinate offsets of the collimator can be initially measured at an arbitrary gantry angle (for example $0^{\circ}$ ) in the reference system, and then applied to any gantry angles in the same manner.

\section{Results}

The isocenters of a linac are defined in this section. The rotation isocenters of the collimator, gantry, and couch will be purely mechanical concepts and they are established in this order, but the linac isocenter and the radiation isocenter definitions include the linac X-ray source position.

At some point during linac installation, the installer has to turn on the radiation beams. In this work, only the photon beams are of consequence. The methods of finding X-ray source position have been reported [20] [21]. We assume that the installer or the physicist has the means of measuring the parameters in Equations (1) and (2), whether using mechanical, optical, radiation, or other methods. Once these parameters and the X-ray source position are determined, the isocenters can be defined from the fundamental concepts.

\subsection{Mechanical Isocenters}

The AOR's of the collimator, gantry, and couch should be established prior to the isocenter definition. An assumption is made that the mechanical motions are accurately reproducible in a short period of time. We propose choosing the source position at $0^{\circ}$ collimator and gantry angles (source position zero), and measuring the trajectory of source position zero when the gantry makes a full rotation. The linac mechanical isocenter or "linac isocenter" is defined as the center of the circle that best fits the trajectory of the source position zero. By this definition, the linac isocenter is a measureable point in space, and it is stationary in the reference frame.

In this work, the collimator rotation isocenter might not be an indispensable term. To be consistent with the AAPM reports, we define the "collimator rotation isocenter" as follows. When the collimator rotates, if the center of the minimal bounding sphere of the trajectory of a point on the collimator AOR is the closest to the gantry AOR, this sphere center is the collimator rotation center. By this definition, the collimator isocenter is not a stationary point, and it is subject to the misalignment shown in Figure 1. Similarly, we propose defining the "gantry rotation isocenter" as follows. When the gantry rotates, if the center of the minimal bounding sphere of the trajectory of a point on the gantry AOR is the closest to the linac isocenter, this sphere center is the gantry rotation isocenter. Thus, the gantry rotation isocenter is a stationary point. The couch is an independent subsystem. For practical purposes, we define the "couch rotation isocenter” the same way as the gantry rotation isocenter: 
As the couch rotates, if the center of the minimal bounding sphere of the trajectory of a point on the couch AOR is closest to the linac isocenter, this sphere center is the couch rotation isocenter. The couch rotation isocenter is also a stationary point in the reference frame.

\subsection{Radiation Isocenter}

So far in our approach, the radiation field has not utilized collimation. In order to define the radiation isocenter, it is now appropriate to consider photon beam collimation. In Nebraska, the above linac isocenter definition does not comply with its regulations to a full extent. Even with the regulations in place, however, medical physicists pay little attention to such kind of definitions. The obvious reason is that collimated radiation fields are routinely used in radiation therapy. From this point of view, Nebraska's isocenter definition is close to clinical reality and can be considered as the radiation isocenter. Therefore, we believe that it is necessary to propose a definition of radiation isocenter that incorporates a surrogate of the "useful beam axis". When the gantry and collimator rotate, the instantaneous $\mathrm{X}$-ray source determined by beam divergence might not remain at a fixed position relative to the gantry. It has been reported that the linac X-ray focal spots of different energies might not be coincident, and that they may shift over time [21]. We also take into account the practical point that the linac jaws, multileaf-collimator (MLC), and circular cones are calibrated against a predetermined point (for example, the projection of the collimator rotation isocenter), which is termed the "center of collimation" in this work. To accommodate these factors, we define the "radiation isocenter" as follows. At any gantry angle, a straight line passes through the instantaneous X-ray source and the center of collimation (note that both are functions of gantry angle). The center of the smallest sphere that all these straight lines can pass through is defined as the radiation isocenter.

The connections among the concepts and the definitions presented above are illustrated in Figure 3.

\subsection{Treatment Target in Radiation Field}

In radiotherapy, the treatment target is expected to be located at a predetermined position. In this section we will consider any point in the target volume, and find the formulae that can calculate its position with respect to a radiation field, which corresponds to arbitrary rotation angles of the three subsystems. The goal of this section is

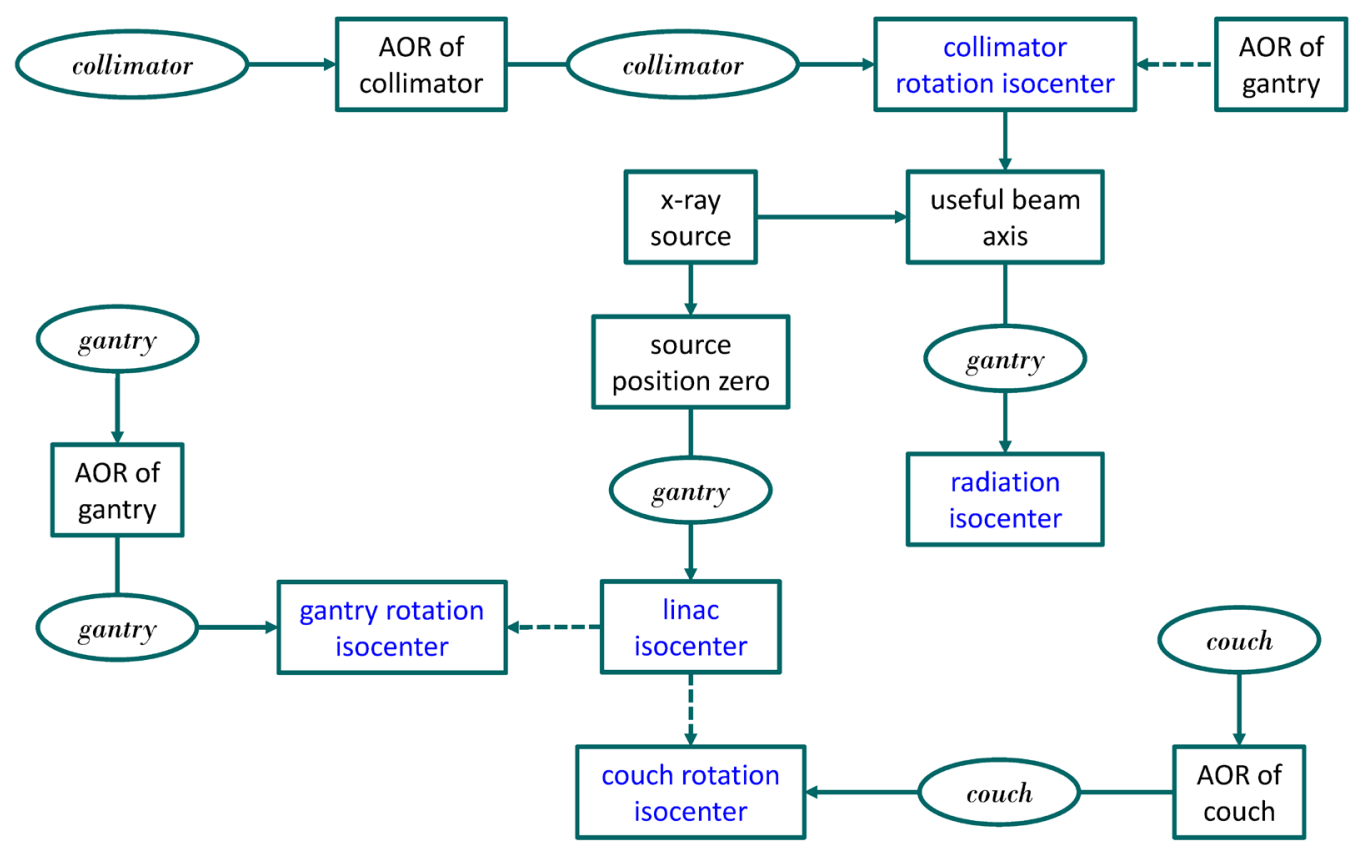

Figure 3. The proposed framework of isocenter definitions. The foundation of this system is the AOR's of the collimator, gantry, and couch. All the AOR's and isocenters are established by the rotation of the subsystems (oval shapes), and the rotation isocenters align with the linac isocenter or the gantry AOR (dashed lines). 
to demonstrate an example of our mathematical model, i.e., to derive the coordinate transformation to the collimator system, because the clinical radiation fields are shaped by the jaws, MLC, or cones. This derivation can directly be used to calculate the position of a target point in a radiation field. Similar treatments should be useful in the isocenter determination and QA processes. For simplicity, the coordinate transformation from the gantry system to the reference system Equation (1) is written as

$$
[X]=M_{G}\left[X_{G}\right]+[G] \text {. }
$$

The transformation from the collimator and couch has the same form except that the symbol $G$ is replaced by $C$ or $T$, respectively. Now we examine the situation in that the collimator, gantry and couch have rotation angles. In reality, the target is placed on the couch. From Equation (3) the transformation from couch system to gantry system is

$$
\left[X_{G}\right]=M_{G}^{-1}\left(M_{T}\left[X_{T}\right]+[T]-[G]\right) .
$$

Rewriting Equation (3) derives the transformation from collimator system to gantry system

$$
\left[X_{G}\right]=M_{C}^{G}\left[X_{C}^{G}\right]+\left[C^{G}\right]=M_{C}\left[X_{C}\right]+[C]-[G] .
$$

The superscript $G$ reflects the fact that collimator system rotates with the gantry. From Equations (4) and (5), it is easy to derive the coordinate transformation from the couch system to the collimator system

$$
\left[X_{C}\right]=M_{C}^{-1}\left(M_{G}^{-1}\left(M_{T}\left[X_{T}\right]+[T]-[G]\right)-[C]+[G]\right) .
$$

\section{Discussion}

In the above sections, we have established the definitions of mechanical isocenters and radiation isocenter. Efforts are made to ensure that these definitions are compatible with the prevailing understanding of the isocenters of a conventional medical linac [1], the AAPM terminologies [2] [5], and occasionally, a regulatory definition. The rotation isocenters are geometric definitions per se. The linac isocenter and radiation isocenters are based on the trajectory of the X-ray source position; therefore, they are not purely mechanical concepts. We recommend that the linac isocenter be used as a spatial reference and the other isocenters be aligned with it. The only exception is the collimator rotation isocenter, which is defined in such a way that it is determined at a single gantry angle, and its position is a function of the gantry angle. The reason for using this definition is to find the useful beam axis at each gantry angle. In this framework, the source to axis distance can still be $100 \mathrm{~cm}$; however, this might not be true for the distance between the X-ray source and the linac isocenter because the definition does not force it to be so. The consequence is that, if a water phantom is aligned with the linac isocenter during output calibration, the nominal source to surface distance will be slightly different from $100 \mathrm{~cm}$. In modern linacs, the resultant dosimetric changes should be usually negligible.

Our work provides medical physicists and linac installers with a rigorous system without ambiguity and circuitous definitions. Among the five isocenters defined above, the radiation isocenter is most relevant to treatment delivery. Because the radiation isocenter is associated with gantry rotation, the position of any point in the target volume relative to the useful beam axis is predictable. This will eliminate some current concerns such as gantry sag which has been integrated into the isocenter definitions in our system. In order to implement these definitions in clinical practice, physicists and engineers have to develop methods that can track the trajectory of any point with sufficient accuracy. Therefore, after the preliminary installation, the linac installer will be able to make mechanical adjustments and line up the isocenters based on accurate measurements that replace the previous trial-and-error techniques.

According to our principle of operability, ancillary devices for beam shaping and target localization are aligned with the isocenters that have been previously determined. From this point of view, those ancillary devices are not appropriate for the measurement of a mechanical or radiation isocenter. In this manuscript, we have developed an isocenter definition system that is independent of measurement techniques. There should be multiple approaches that can utilize this system to determine the isocenters of a medical linac. The pursuit of a rigorous isocenter definition system will result in the expense of complicated measurements. Because the subsystem rotations are involved, we anticipate the measurements to be tedious and not suitable for periodic QA tests. Another limitation we have realized is that, in this system the isocenters are defined in the linac coordinate sys- 
tem. When utilizing our system, a physicist might need a surrogate to locate an isocenter in the reference system. It is unlikely that the resources necessary for the isocenter measurements are accessible by all the clinical physicists. The majority of physicists have to rely on commercial products or some simplified approaches. In reality, a subsample of full measurements might provide acceptable accuracy and be used in periodic QA. Currently we are working on an approach that utilizes the definitions and methods proposed in this manuscript to measure the isocenters in three-dimensional space. It is expected that in the future, medical physicists will be able to quantify the rotation isocenters and the coincidence of radiation and mechanical isocenters during annual QA [2].

Rigorous definitions help physicists understand clinical procedures where an isocenter plays a role. Now we exemplify using analysis of the traditional frame-based SRS on a medical linac, in which the treatment target must be precisely positioned at the radiation isocenter. Because an isocenter is invisible, lasers have to be used as surrogates. Conventionally, the lasers are aligned to the linac isocenter through a mechanical approach, thus they approximately represent the linac isocenter. Before the SRS procedure, the Winston-Lutz test is performed using a ball bearing phantom [4]. The purpose of this test is to verify that the treatment target can be aligned with the radiation isocenter within a preset tolerance. Whether or not the ball bearing phantom is aligned to the lasers prior to the test, the isocenter presented by the lasers is different from the intended isocenter for treatment delivery. Distinguishing the two isocenters is critical to the SRS procedure because if the target is aligned to the mechanical isocenter, the discrepancy results in a residual setup error. Thus, the lasers must represent the radiation isocenter rather than the mechanical isocenter.

One may ask, is the Winston-Lutz test an acceptable method for radiation isocenter measurement? In our opinion, this is doubtful. Firstly, the Winston-Lutz test takes subsamples of the measurements that are necessary to determine the optimal treatment target position; Secondly and more importantly, the collimated radiation fields that the Winston-Lutz test employs do not necessarily provide the useful beam axis for any angle combination. If a Winston-Lutz type approach is used to determine an optimal treatment target location, it should not be considered the exact radiation isocenter. We can use the Elekta "flexmap" [22] approach as an example to illustrate our opinion. The flexmap approach contains isocenter verification using a ball bearing phantom. The method is similar to the conventional Winston-Lutz test. Briefly, a ball bearing phantom is placed close to the linac isocenter. At each of the $0^{\circ}, 90^{\circ}, 180^{\circ}$ and $270^{\circ}$ gantry angles, two MV images are taken using $10 \mathrm{~cm} \times 10 \mathrm{~cm}$ open fields at $0^{\circ}$ and $180^{\circ}$ collimator angles, respectively (Figure 4). There are eight images all together, from which the positional difference between the ball bearing projection and the field center is calculated for the left-right, superior-inferior, and anterior-posterior directions. In practice, one can move the ball bearing to locate its center at the position of the average center of the eight open fields, and then bring the lasers to this location. This procedure is analogous to the Winston-Lutz test (without couch rotation), and in principle it does not determine a rigorous radiation isocenter as defined in this work.

Currently, image guided frameless SRS procedures are replacing frame-based SRS. We would like to point out that relative to the subsystems of a linac, image guidance systems are secondary in nature, whether or not they are integrated into the mechanical structure of the linac. Although image guidance is increasingly used in radiotherapy, this fact does not compromise the importance of appropriate isocenter definitions. Nowadays some image guidance systems still rely on the lasers for calibration - their calibration phantoms are aligned to the lasers. Even if the lasers are no longer essential for patient setup when image guidance is in place, the discrepancy between the radiation isocenter and the imaging isocenter becomes crucial [23]. It is predicted that more patients will undergo stereotactic radiosurgery or stereotactic body radiosurgery. The demand of precise dose delivery in these procedures makes the clear identification of isocenters more critical than ever.

\section{Conclusion}

In this manuscript, a complete system of isocenter definitions is proposed for medical linacs. Minimal bounding sphere and coordinate transformation are the fundamental mathematical tools in the system. The following terms have been defined: linac isocenter, collimator rotation isocenter, gantry rotation isocenter, couch rotation isocenter, and radiation isocenter. These definitions are derived from the axes of rotation of the linac subsystemscollimator, gantry, and couch through operable procedures. This work provides the clinical physicists and linac engineers with a framework to perform measurements and quality assurance tests pertinent to the isocenters and radiation fields of a medical linear accelerator. 


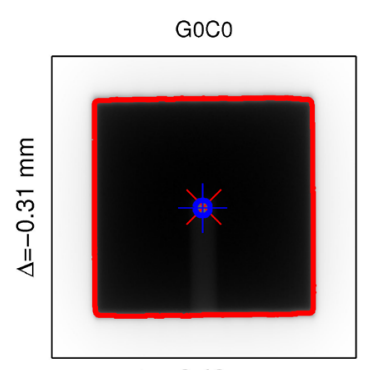

$\Delta=-0.40 \mathrm{~mm}$

Total $\Delta=0.51 \mathrm{~mm}$

G180C0

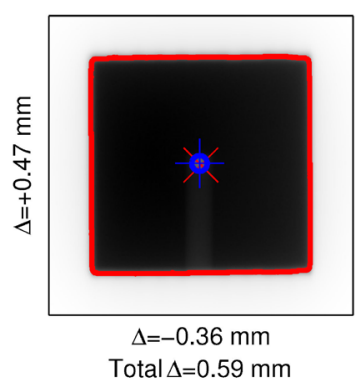

GoC180

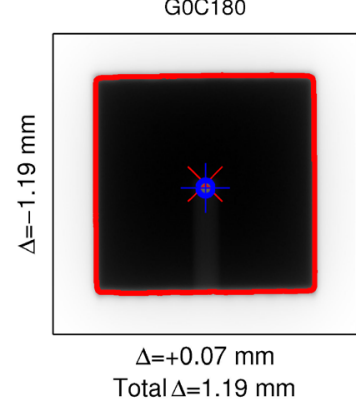

G180C180

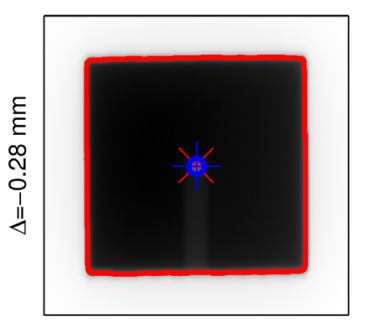

$\Delta=+0.10 \mathrm{~mm}$ Total $\Delta=0.30 \mathrm{~mm}$

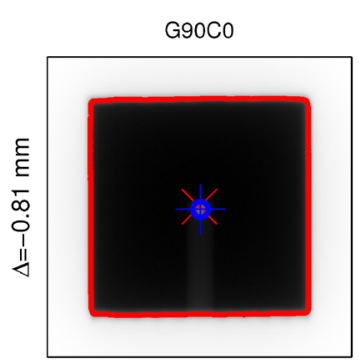

$\Delta=+0.32 \mathrm{~mm}$

Total $\Delta=0.87 \mathrm{~mm}$

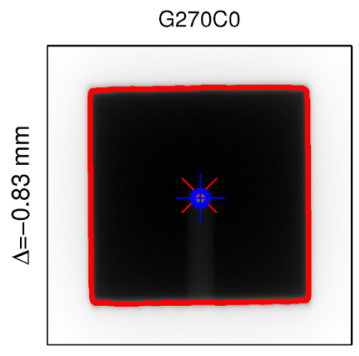

$\Delta=+0.29 \mathrm{~mm}$ Total $\Delta=0.88 \mathrm{~mm}$

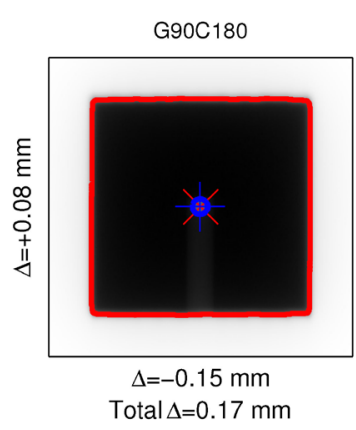

G270C180

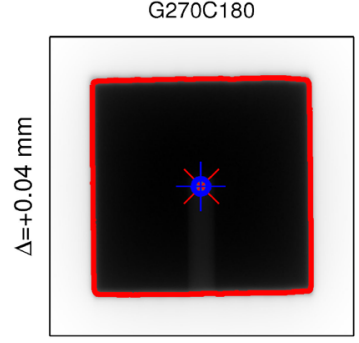

$\Delta=-0.17 \mathrm{~mm}$ Total $\Delta=0.17 \mathrm{~mm}$

Figure 4. The Elekta flexmap approach of localizing the optimal target position (the ball bearing, blue circle). The images are independently verified with DoseLab (Mobius Medical Systems, Houston, TX). Shown in each image are the ball bearing center (blue cross) and the optimal target position (red cross), and the coordinate offsets of the ball bearing in each radiation field.

\section{Acknowledgements}

The authors are grateful to the medical physics colleagues and linac engineers who shared their insights on this topic. Mr. Chuck Rhode acquired the images shown in Figure 4. Dr. Xiaofeng Zhu reviewed this manuscript and provided invaluable critiques. Dr. Vivek Verma edited this manuscript.

\section{References}

[1] Nath, R., Biggs, P.J., Bova, F.J., Ling, C.C., Purdy, J.A., van de Geijn, J. and Weinhous, M.S. (1994) AAPM Code of Practice for Radiotherapy Accelerators: Report of AAPM Radiation Therapy Task Group No. 45. Medical Physics, 21, 1093-1121. http://dx.doi.org/10.1118/1.597398

[2] Klein, E.E., Hanley, J., Bayouth, J., Yin, F.F., Simon, W., Dresser, S., Serago, C., Aguirre, F., Ma, L., Arjomandy, B., Liu, C., Sandin, C. and Holmes, T., Task Group 142, American Association of Physicists in Medicine (2009) Task Group 142 Report: Quality Assurance of Medical Accelerators. Medical Physics, 36, 4197-4212. http://dx.doi.org/10.1118/1.3190392

[3] Huang, Y., Zhao, B., Chetty, I.J., Brown, S., Gordon, J. and Wen, N. (2015) Targeting Accuracy of Image-Guided Radiosurgery for Intracranial Lesions: A Comparison across Multiple Linear Accelerator Platforms. Technology in Cancer Research \& Treatment.

[4] Lutz, W., Winston, K.R. and Maleki, N. (1988) A System for Stereotactic Radiosurgery with a Linear Accelerator. International Journal of Radiation Oncology* Biology* Physics, 14, 373-381. http://dx.doi.org/10.1016/0360-3016(88)90446-4

[5] Kutcher, G.J., Coia, L., Gillin, M., Hanson, W.F., Leibel, S., Morton, R.J., Palta, J.R., Purdy, J.A., Reinstein, L.E., Svensson, G.K., et al. (1994) Comprehensive QA for Radiation Oncology: Report of AAPM Radiation Therapy Committee Task Group 40. Medical Physics, 21, 581-618. http://dx.doi.org/10.1118/1.597316

[6] Rowshanfarzad, P., Sabet, M., O’Connor, D.J. and Greer, P.B. (2011) Isocenter Verification for Linac-Based Stereotactic Radiation Therapy: Review of Principles and Techniques. Journal of Applied Clinical Medical Physics, 12, 185-195.

[7] Khan, F.M. (1994) The Physics of Radiation Therapy. 2nd Edition, Lippincott, Williams \& Wilkins, Philadelphia, 518.

[8] Khan, F.M. (2009) The Physics of Radiation Therapy. 4th Edition, Lippincott, Williams \& Wilkins, Philadelphia, 145. 
[9] Du, W., Gao, S., Wang, X. and Kudchadker, R.J. (2012) Quantifying the Gantry Sag on Linear Accelerators and Introducing an MLC-Based Compensation Strategy. Medical Physics, 39, 2156-2162. http://dx.doi.org/10.1118/1.3697528

[10] Rowshanfarzad, P., McGarry, C.K., Barnes, M.P., Sabet, M. and Ebert, M.A. (2014) An EPID-Based Method for Comprehensive Verification of Gantry, EPID and the MLC Carriage Positional Accuracy in Varian Linacs during Arc Treatments. Radiation Oncology, 9, 249. http://dx.doi.org/10.1186/s13014-014-0249-8

[11] Schiefer, H., Ingulfsen, N., Kluckert, J., Peters, S. and Plasswilm, L. (2015) Measurements of Isocenter Path Characteristics of the Gantry Rotation Axis with a Smartphone Application. Medical Physics, 42, 1184-1192. http://dx.doi.org/10.1118/1.4906248

[12] González, A., Castro, I. and Martínez, J.A. (2004) A Procedure to Determine the Radiation Isocenter Size in a Linear Accelerator. Medical Physics, 31, 1489-1493. http://dx.doi.org/10.1118/1.1755491

[13] Rosca, F., Lorenz, F., Hacker, F.L., Chin, L.M., Ramakrishna, N. and Zygmanski, P. (2006) An MLC-Based Linac QA Procedure for the Characterization of Radiation Isocenter and Room Lasers’ Position. Medical Physics, 33, 1780-1787. http://dx.doi.org/10.1118/1.2198171

[14] Du, W. and Gao, S. (2011) Measuring the Wobble of Radiation Field Centers during Gantry Rotation and Collimator Movement on a Linear Accelerator. Medical Physics, 38, 4575-4578. http://dx.doi.org/10.1118/1.3609098

[15] Moyers, M.F. and Lesyna, W. (2004) Isocenter Characteristics of an External Ring Proton Gantry. International Journal of Radiation Oncology*Biology*Physics, 60, 1622-1630. http://dx.doi.org/10.1016/j.ijrobp.2004.08.052

[16] Welzl, E. (1991) Smallest Enclosing Disks (Balls and Ellipsoids). In: Maurer, H., Ed., New Results and New Trends in Computer Science, Lecture Notes in Computer Science, Vol. 555, Springer, New York, 359-370. http://dx.doi.org/10.1007/bfb0038202

[17] Larsson, T. (2008) Fast and Tight Fitting Bounding Spheres. Proceedings of the Annual SIGRAD Conference, Vol. 34 Stockholm, 27-30.

[18] Zhang, M., Zaider, M., Worman, M. and Cohen, G. (2004) On the Question of 3D Seed Reconstruction in Prostate Brachytherapy: The Determination of X-Ray Source and Film Locations. Physics in Medicine and Biology, 49, N335N345.

[19] Siddon, R.L. (1981) Solution to Treatment Planning Problems Using Coordinate Transformations. Medical Physics, 8, 766-774. http://dx.doi.org/10.1118/1.594853

[20] Tatcher, M. and Bjärngard, B. (1992) Head-Scatter Factors and Effective X-Ray Source Positions in a 25-MV Linear Accelerator. Medical Physics, 19, 685-686. http://dx.doi.org/10.1118/1.596811

[21] Jaffray, D.A., Battista, J.J., Fenster, A. and Munro, P. (1993) X-Ray Sources of Medical Linear Accelerators: Focal and Extra-Focal Radiation. Medical Physics, 20, 1417-1427. http://dx.doi.org/10.1118/1.597106

[22] Sharpe, M.B., Moseley, D.J., Purdie, T.G., Islam, M., Siewerdsen, J.H. and Jaffray, D.A. (2006) The Stability of Mechanical Calibration for a kV Cone Beam Computed Tomography System Integrated with Linear Accelerator. Medical Physics, 33, 136-144. http://dx.doi.org/10.1118/1.2143141

[23] Tideman Arp, D. and Carl J. (2012) EXACTRAC X-Ray and Beam Isocenters-What's the Difference? Medical Physics, 39, 1418-1423. http://dx.doi.org/10.1118/1.3685581 